\title{
Oscillatory and Asymptotic Behaviour of Solutions of Two Nonlinear Dimensional Difference Systems
}

\author{
G. Saraswathi' ${ }^{1}$ P. Sumathi ${ }^{2}$ \\ ${ }^{1}$ Department of Mathematics, Chellammal Women's College, Chennai, India \\ ${ }^{2}$ Department of Mathematics, C. Kandaswami Naidu College for Men, Chennai, India \\ Email: ganesan saraswathi@yahoo.co.in, sumathipaul@gmail.com
}

How to cite this paper: Saraswathi, G. and Sumathi, P. (2019) Oscillatory and Asymptotic Behaviour of Solutions of Two Nonlinear Dimensional Difference Systems. Journal of Applied Mathematics and Physics, 7, 1001-1011. https://doi.org/10.4236/jamp.2019.74067

Received: February 20, 2019

Accepted: April 27, 2019

Published: April 30, 2019

Copyright $\odot 2019$ by author(s) and Scientific Research Publishing Inc. This work is licensed under the Creative Commons Attribution International License (CC BY 4.0).

http://creativecommons.org/licenses/by/4.0/

\begin{abstract}
This paper deals with the some oscillation criteria for the two dimensional difference system of the form: $\begin{aligned} & \Delta x_{n}=b_{n} y_{n}^{\alpha} \\ & \Delta y_{n}=-a_{n} x_{n}^{\beta}, n_{0} \in N=1,2,3, \ldots\end{aligned}$. Examples il-
\end{abstract} lustrating the results are inserted.

\section{Keywords}

Asymptotic, Two-Dimensional Difference Systems

\section{Introduction}

Consider a nonlinear two dimensional difference system of the form

$$
\begin{aligned}
& \Delta x_{n}=b_{n} y_{n}^{\alpha} \\
& \Delta y_{n}=-a_{n} x_{n}^{\beta}, n_{0} \in N=1,2,3, \cdots
\end{aligned}
$$

where $\left\{a_{n}\right\}$ and $\left\{b_{n}\right\}$ are real sequences and $n \in N\left(n_{0}\right), \alpha$ and $\beta$ are ratio of odd positive integers.

By a solution of Equation (1.1), we mean a real sequence $\left\{x_{n}\right\}$ which is defined for all $n \geq n_{0}$ and satisfies Equation (1.1) for all $n \in N\left(n_{0}\right)$.

In the last few decades there has been an increasing interest in obtaining necessary and sufficient conditions for the oscillation and nonoscillation of two dimensional difference equation. See for example [1]-[10] [11] and the references cited therein.

Further it will be assumed that $\left\{b_{n}\right\}$ is non-negative for all $n \geq n_{0}$, $u^{\beta}-v^{\beta}=\frac{u^{\beta}-v^{\beta}}{u-v}(u-v)$ for all $u, v$. 
The oscillation criteria for system (1.1), when

$$
\sum_{s=n_{0}}^{\infty} a_{s}=\infty
$$

studied in [12]. Therefore in this paper we consider the other case that is

$$
\sum_{s=n_{0}}^{\infty} a_{s}<\infty
$$

and investigated the oscillatory behaviour of solutions of the system (1.1). Hence the results obtained in this paper complement to that of in [12].

We may introduce the function $A_{n}$ defined by

$$
A_{n}=\sum_{s=n+1}^{\infty} a_{s}, n \in N\left(n_{0}\right)
$$

Throughout this paper condition (1.2) is tacitly assumed; $A_{n}$ always denotes the function defined by (1.3).

In Section 2, we establish necessary and sufficient conditions for the system (1.1) to have solutions which behave asymptotically like nonzero constants or linear functions and in Section 3, we present criteria for the oscillation of all solutions of the system (1.1). Examples are inserted to illustrate some of the results in Section 4.

\section{Existence of Bounded/Unbounded Solutions}

In this section first we obtain necessary and sufficient conditions for the system (1.1) to have solutions which behave asymptotically like nonzero constants.

Theorem 2.1. If

$$
\sum_{n=n_{0}}^{\infty}\left|A_{n}\right|^{\alpha}<\infty
$$

and

$$
\sum_{n=n_{0}}^{\infty} B_{n}^{\alpha}<\infty
$$

are satisfied, then for any constant $c \neq 0$, system (1.1) has a solution $\left(\left\{x_{n}\right\},\left\{y_{n}\right\}\right)$. such that

$$
\begin{gathered}
x_{n}=c+o\left(\sum_{s=n}^{\infty}\left(\left|A_{s}\right|^{\alpha}+B_{s}^{\alpha}\right)\right) \\
y_{n}=o\left(\left|A_{n}\right|+B_{n}\right)
\end{gathered}
$$

as $n \rightarrow \infty$, where

$$
B_{n}=\sum_{s=n+1}^{\infty}\left|A_{s}\right|^{\alpha+1}
$$

Proof. We may assume without loss of generality that $c>0$. Let

$$
\mu=\max \left\{u^{\beta} ; \frac{c}{2} \leq u \leq \frac{3 c}{2}\right\}
$$




$$
\delta=\max \left\{\frac{u^{\beta}-v^{\beta}}{u-v} ; \frac{c}{2} \leq u, v \leq \frac{3 c}{2}\right\}
$$

choose $\lambda>0$, so that

$$
M \delta(\mu)^{\alpha}=\frac{\lambda}{2}
$$

and let $N \in \mathbb{N}\left(n_{0}\right)$ be large enough such that

$$
\begin{gathered}
M(\mu)^{\alpha} \sum_{n=N}^{\infty}\left|A_{n}\right|^{\alpha} \leq \frac{c}{4} \\
M(\lambda)^{\alpha} \sum_{n=N}^{\infty} B_{n}^{\alpha} \leq \frac{c}{4}
\end{gathered}
$$

and

$$
M \delta(\lambda)^{\alpha} \sum_{n=N}^{\infty} B_{n}^{\alpha} \leq \frac{\lambda}{2}
$$

Let $B$ be the space of all real sequences $y=\left\{y_{n}\right\}, n \geq N$ with the topology of pointwise convergence. We now define $X$ to be the set of sequences $x \in B$. such that

$$
\left|x_{n}-c\right| \leq \frac{c}{2}, n \geq N
$$

and

$$
\left|x_{n_{1}}-x_{n_{2}}\right| \leq M\left((\mu)^{\alpha}\left|\bar{A}_{n}\right|^{\alpha}+(\lambda)^{\alpha} B_{N}^{\alpha}\right)\left|n_{1}-n_{2}\right|, n_{1}, n_{2} \geq N
$$

where $\left|\bar{A}_{N}\right|=\sup \left(\left|A_{n}\right|: n \geq N\right)$ and define $Y$ to be the set of sequences $y \in B$. Such that

$$
\left|y_{n}\right| \leq \mu\left|A_{n}\right|+\lambda B_{n}, n \geq N
$$

Let $T_{1}$ and $T_{2}$ denote the mappings from $X \times Y \rightarrow B$ defined by

$$
T_{1}(x, y)_{n}=C-\sum_{s=n}^{\infty} b_{s} y_{s}^{\alpha} n>N
$$

and

$$
T_{2}(x, y)_{n}=A_{n} x_{n+1}^{\beta}+\sum_{s=n}^{\infty} A_{s} b_{s} y_{s}^{\alpha} \frac{x_{s+1}^{\beta}-x_{s}^{\beta}}{x_{s+1}-x_{s}}, n \geq N .
$$

Finally define $T: X \times Y \rightarrow B \times B$ by

$$
T(x, y)=\left(T_{1}(x, y), T_{2}(x, y)\right),(x, y) \in X \times Y
$$

Clearly $X \times Y$ is a bounded, closed and convex subset of $B \times B$.

First we show that $T$ maps $X \times Y$ into itself. Let $(x, y) \in X \times Y$. From (2.11), we have

$$
y_{n}^{\alpha} \leq(\mu)^{\alpha}\left|A_{n}\right|^{\alpha}+(\lambda)^{\alpha} B_{n}^{\alpha}, n \geq N .
$$

and so, using (2.6) and (2.7), we see that 


$$
\begin{aligned}
\sum_{s=n}^{\infty} b_{s} y_{s}^{\alpha} & \leq M \sum_{s=n}^{\infty}\left((\mu)^{\alpha}\left|A_{s}\right|^{\alpha}+(\lambda)^{\alpha}\left(B_{s}\right)^{\alpha}\right) \\
& \leq M(\mu)^{\alpha} \sum_{s=n}^{\infty}\left|A_{s}\right|^{\alpha}+M(\lambda)^{\alpha} \sum_{s=n}^{\infty} B_{s}^{\alpha} \\
& \leq \frac{C}{4}+\frac{C}{4}=\frac{c}{2}, n \geq N .
\end{aligned}
$$

Now from (2.12) it follows that

$$
\left|T_{1}(x, y)_{n}-c\right| \leq \frac{c}{2}, n \geq N .
$$

Moreover,

$$
\begin{aligned}
& \left|T_{1}(x, y)_{n_{1}}-T_{1}(x, y)_{n_{2}}\right| \\
& =\left|\sum_{s=n_{1}}^{n_{2}-1} b_{s} y_{s}^{\alpha}\right| \leq\left. M\left|\sum_{s=n_{1}}^{n_{2}-1}(\mu)^{\alpha}\right| A_{s}\right|^{\alpha}+(\lambda)^{\alpha} B_{s}^{\alpha} \mid \\
& \leq M\left((\mu)^{\alpha}\left|A_{N}\right|^{\alpha}+(\lambda)^{\alpha} B_{N}^{\alpha}\right)\left|n_{1}-n_{2}\right|
\end{aligned}
$$

for $n_{1}, n_{2} \geq N$. This implies that $T_{1}(x, y) \in X$. Next from (2.13), we have

$$
\begin{aligned}
\left|T_{2}(x, y)_{n}\right| & \leq \mu\left|A_{n}\right|+M \delta \sum_{s=n+1}^{\infty}\left|A_{s}\right|\left((\mu)^{\alpha}\left|A_{s}\right|^{\alpha}+(\lambda)^{\alpha} B_{s}^{\alpha}\right) \\
& \leq \mu\left|A_{n}\right|+M \delta(\mu)^{\alpha} \sum_{s=n+1}^{\infty}\left|A_{s}\right|^{\alpha+1}+M \delta(\lambda)^{\alpha} \sum_{s=n+1}^{\infty}\left|A_{s}\right| B_{s}^{\alpha} \\
& \leq \mu\left|A_{n}\right|+\frac{\lambda}{2} B_{n}+M \delta(\lambda)^{\alpha} B_{n}\left(\sum_{s=n+1}^{\infty} B_{s}^{\alpha}\right) \\
& \leq \mu\left|A_{n}\right|+\lambda B_{n}, n \geq N .
\end{aligned}
$$

where conditions (2.5), (2.7) and (2.10) have been used. Thus $T_{2}(x, y) \in Y$. Hence $T(x, y) \in X \times Y$ as desired.

Now let $(x, y)=\left(x_{n}, y_{n}\right) \in X \times Y$ and for each $i=1,2, \cdots$. Let $\left(x^{i}, y^{i}\right)=\left(x_{n}^{i}, y_{n}^{i}\right)$ be a sequence in $X \times Y$. Such that $\lim _{i \rightarrow \infty}\left\|\left(x^{i}, y^{i}\right)-(x, y)\right\|=0$. Then a straight forward argument $\lim _{i \rightarrow \infty}\left\|T\left(x_{n}^{i}, y_{n}^{i}\right)-T\left(x_{n}, y_{n}\right)\right\|=0$ and hence $T$ is continuous.

Finally, in order to apply Schauder-Tychonoff fixed point theorem, we need to show that $T(X \times Y)$ is relatively compact in $B \times B$. In view of recent result of cheng and patula [8] it suffices to show that $T(X \times Y)$ is uniformly cauchy in $B \times B$. To prove this, it is enough to show that $T_{1}(X \times Y)$ and $T_{2}(X \times Y)$ are uniformly cauchy in $B$. To this end, let $(x, y)=\left(x_{n}, y_{n}\right) \in X \times Y$ and observe that for any $k>n \geq N$, we have

$$
\left|T_{1}(x, y)_{k}-T_{1}(x, y)_{n}\right| \leq M \sum_{s=n+1}^{\infty}\left((\mu)^{\alpha}\left|A_{s}\right|^{\alpha}+(\lambda)^{\alpha} B_{s}^{\alpha}\right)
$$

and

$$
\left|T_{2}(x, y)_{k}-T_{2}(x, y)_{n}\right|=2 \mu\left|A_{n}\right|+M \delta \sum_{s=n+1}^{\infty}\left|A_{s}\right|\left((\mu)^{\alpha}\left|A_{s}\right|^{\alpha}+(\lambda)^{\alpha} B_{s}^{\alpha}\right)
$$

It is now clear that for a given $\epsilon>0$, we can choose $N_{1} \geq N$, such that 
$k>n \geq N_{1}$, imply $\left|T_{1}(x, y)_{k}-T_{1}(x, y)_{n}\right|<\epsilon$ and $\left|T_{2}(x, y)_{k}-T_{2}(x, y)_{n}\right|<\epsilon$. Thus $T_{1}(X \times Y)$ and $T_{2}(X \times Y)$ are uniformly cauchy and so $T(X \times Y)$ is uniformly cauchy. Thus $T(X \times Y)$ is relatively compact.

Therefore by Schauder-Tychonoff fixed point theorem, there is an element $(x, y) \in X \times Y$ such that $T(x, y)=(x, y)$. From (2.12), (2.13) and (2.14)

$$
\begin{gathered}
x_{n}=c-\sum_{s=n}^{\infty} b_{s} y_{s}^{\alpha} \\
y_{n}=A_{n} x_{n+1}^{\beta}+\sum_{s=n+1}^{\infty} A_{s} b_{s} y_{s}^{\alpha} \frac{x_{s+1}^{\beta}-x_{s}^{\beta}}{x_{s+1}-x_{s}}
\end{gathered}
$$

From (2.15) and (2.16), we see that $\left(\left\{x_{n}\right\},\left\{y_{n}\right\}\right)$ is a solution of then system (1.1) with the properties (2.3) and (2.4). This completes the proof of the theorem.

Corollary 2.2. Assume (2.1) and (2.2) are satisfied. Then for any $c \neq 0$ system (1.1) has a nonoscillatory solution $\left(\left\{x_{n}\right\},\left\{y_{n}\right\}\right)$ such that

$$
\begin{aligned}
& x_{n}=c+o(1), \\
& y_{n}=o(1)
\end{aligned}
$$

as $n \rightarrow \infty$. The proof is left to the reader.

Before stating and proving our next results, we give a lemma which is concerned with the nonoscillatory solution of (1.1).

Lemma 2.3. Let $\left(\left\{x_{n}\right\},\left\{y_{n}\right\}\right)$ be a solution of (1.1) for $n \geq N \in \mathbb{N}\left(n_{0}\right)$ with $x_{n}>0$ for all $n \geq N$. Then

$$
\sum_{i=N}^{\infty} \frac{b_{i} y_{i}^{\alpha+1} \frac{x_{n+1}^{\beta}-x_{n}^{\beta}}{x_{n+1}-x_{n}}}{x_{i+1}^{\beta} x_{i}^{\beta}}<\infty
$$

and

$$
\frac{y_{n}}{x_{n+1}^{\beta}}=\theta+A_{n}+\sum_{i=n+1}^{\infty} \frac{b_{i} y_{i}^{\alpha+1} \frac{x_{i+1}^{\beta}-x_{i}^{\beta}}{x_{i+1}-x_{i}}}{x_{i+1}^{\beta} x_{i}^{\beta}}
$$

for $n \geq N$, where $\theta$ is a nonnegative constant.

This lemma has been proved by Graef and Thandapani [3] and is very useful in the following theorems. In our next theorem, we establish a necessary condition for the system (1.1) to have nonoscillatory solution satisfying condition (2.17).

Theorem 2.4. Assume that $A_{n} \geq 0$ for all $n \in \mathbb{N}\left(n_{0}\right)$. Then a necessary condition for the system (1.1) to have a nonoscillatory solution $\left(\left\{x_{n}\right\},\left\{y_{n}\right\}\right)$ satisfying (2.17) is that

$$
\sum_{n=n_{0}}^{\infty} b_{n} A_{n}^{\alpha}<\infty \text { and } \sum_{n=n_{0}}^{\infty} b_{n}\left(\sum_{s=n+1}^{\infty} b_{s} A_{s}^{\alpha+1}\right)^{\alpha}<\infty .
$$

Proof. Let $\left(x_{n}, y_{n}\right)$ be a nonoscillatory solution of the system (1.1) for $n \in \mathbb{N}\left(n_{0}\right)$. Since $b_{n}$ is not identically zero for $n \in \mathbb{N}\left(n_{0}\right)$. Hence $x_{n}$ is 
nonoscillatory, without loss of generality, we may assume that $x_{n}$ is eventually positive for $n \in \mathbb{N}\left(n_{0}\right)$. From Lemma 2.3, we have $y_{n}>0$ for $n \geq N \geq n_{0}$ and

$$
y_{n} \geq A_{n} x_{n+1}^{\beta}
$$

and

$$
y_{n} \geq x_{n+1}^{\beta} \sum_{i=n+1}^{\infty} \frac{b_{i} y_{i}^{\alpha+1} \frac{x_{i+1}^{\beta}-x_{i}^{\beta}}{x_{i+1}-x_{i}}}{x_{i}^{\beta} x_{i+1}^{\beta}}, n \geq N .
$$

Since $A_{n} \rightarrow 0$ as $n \rightarrow \infty$, from the first equation of system (1.1), we obtain for $n \geq N$,

$$
\Delta x_{n} \geq b_{n}\left(A_{n} x_{n+1}^{\beta}\right)^{\alpha} \geq b_{n}\left(A_{n}\right)^{\alpha}\left(x_{n+1}^{\beta}\right)^{\alpha}
$$

and hence

$$
\sum_{s=N}^{n-1} b_{s} A_{s}^{\alpha} \leq \sum_{s=N}^{n-1} \frac{\Delta x_{s}}{x_{s+1}^{\alpha \beta}}
$$

Define $\gamma(t)=x_{n}+(t-n) \Delta x_{n}, n \leq t \leq n+1$. If $\Delta x_{n} \geq 0$, then $x_{n} \leq \gamma(t) \leq x_{n+1}$ and

$$
\frac{\Delta x_{n}}{x_{n+1}^{\alpha \beta}} \leq \frac{\gamma^{\prime}(t)}{\gamma(t)^{\alpha \beta}} \leq \frac{\Delta x_{n}}{x_{n}^{\alpha \beta}}
$$

If $\Delta x_{n}<0$, then $x_{n+1} \leq \gamma(t) \leq x_{n}$ and (2.23) again holds. From (2.22) and (2.23), we obtain

$$
\sum_{s=N}^{n-1} b_{s} A_{s}^{\alpha} \leq \int_{x_{N}}^{x_{n}} \frac{\mathrm{d} s}{(s)^{\alpha \beta}}
$$

which in view of the boundedness of $x_{n}$ implies that

$$
\sum_{n=N}^{\infty} b_{n} A_{n}^{\alpha}<\infty .
$$

From the second inequality of (2.21) and the following inequality

$$
\frac{b_{n} y_{n}^{\alpha+1} \frac{x_{n+1}^{\beta}-x_{n}^{\beta}}{x_{n+1}-x_{n}}}{x_{n}^{\beta} x_{n+1}^{\beta}} \geq \frac{b_{n} A_{n}\left(x_{n+1}\right)^{\beta}\left(A_{n}\right)^{\alpha}\left(x_{n+1}\right)^{\alpha \beta} \frac{x_{n+1}^{\beta}-x_{n}^{\beta}}{x_{n+1}-x_{n}}}{x_{n}^{\beta} x_{n+1}^{\beta}} \geq d b_{n} A_{n}^{\alpha+1}, n \geq N .
$$

where " $d$ " being the constant, we see that

$$
d \sum_{s=n+1}^{\infty} b_{s} A_{s}^{\alpha+1} \leq \frac{y_{n}}{x_{n+1}^{\beta}}
$$

Since $\sum_{s=n+1}^{\infty} b_{s} A_{s}^{\alpha+1} \rightarrow 0$ as $n \rightarrow \infty$, from the first equation of system (1.1), we obtain for $n \geq N$

$$
\begin{aligned}
\Delta x_{n} & \geq b_{n}\left(d\left(x_{n+1}^{\beta}\right) \sum_{s=n+1}^{\infty} b_{s} A_{s}^{\alpha+1}\right)^{\alpha} \\
& \geq b_{n}(d)^{\alpha}\left(x_{n+1}^{\beta}\right)^{\alpha}\left(\sum_{s=n+1}^{\infty} b_{s} A_{s}^{\alpha+1}\right)^{\alpha}
\end{aligned}
$$


Hence

$$
(d)^{\alpha} \sum_{s=N}^{n-1} b_{s}\left(\sum_{i=s+1}^{\infty} b_{i} A_{i}^{\alpha+1}\right)^{\alpha} \leq \sum_{s=N}^{n+1} \frac{\Delta x_{s}}{\left(x_{s+1}^{\beta}\right)^{\alpha}} \leq \int_{x_{N}}^{x_{n}} \frac{\mathrm{d} s}{(s)^{\alpha \beta}}
$$

which in view of boundedness of $x_{n}$, implies that

$$
\sum_{n=N}^{\infty} b_{n}\left(\sum_{i=n+1}^{\infty} b_{i} A_{i}^{\alpha+1}\right)^{\alpha}<\infty
$$

The inequalities (2.24) and (2.25) clearly imply (2.20). This completes the proof.

we conclude this section with the following theorem which gives a necessary condition for the system (1.1) to have a nonoscillatory solution of the form

$$
x_{n}=n(c+o(1)), y_{n}=c+o(1), \text { as } n \rightarrow \infty .
$$

Theorem 2.5. Assume $A_{n} \geq 0$ for $n \in \mathbb{N}\left(n_{0}\right)$. The system (1.1) has a solution of the type (2.26) for some $c \neq 0$, then

$$
\sum_{n=n_{0}}^{\infty}\left|k_{1}^{\alpha \beta}(n+1)^{\alpha \beta}\right| \frac{k_{2}^{\beta}(n+1)^{\beta}-k_{2}^{\beta} n^{\beta}}{k_{2}} A_{n}^{\alpha+1}<\infty
$$

for some $k_{1}, k_{2} \neq 0$.

Proof. Let $\left(x_{n}, y_{n}\right)$ be a solution of (1.1) satisfying (2.26). we may assume $c>0$. Then there is an integer $N \in \mathbb{N}\left(n_{0}\right)$. such that

$$
\frac{c n}{2} \leq x_{n} \leq 2 c n \text { for } n \geq N \text {. }
$$

From Lemma 2.2, it follows that

$$
y_{n}=\theta x_{n+1}^{\beta}+A_{n} x_{n+1}^{\beta}+x_{n+1}^{\beta} \sum_{s=n+1}^{\infty} \frac{b_{s} y_{s}^{\alpha+1} \frac{x_{s+1}^{\beta}-x_{s}^{\beta}}{x_{s+1}-x_{s}}}{x_{s}^{\beta} x_{s+1}^{\beta}}
$$

for $n \geq N$, where $\theta$ is a nonnegative constant. Also from the second equation of (1.1), we have

$$
y_{n}=\beta+A_{n} x_{n+1}^{\beta}-\sum_{s=N}^{n} A_{s} b_{s} y_{s}^{\alpha} \frac{\chi_{s+1}^{\beta}-x_{s}^{\beta}}{x_{s+1}-x_{s}}
$$

where $\beta=y_{N-1}-A_{N-1} x_{N}^{\beta}$ combining (2.28) and (2.29), we have

$$
\theta x_{n+1}^{\beta}+x_{n+1}^{\beta} \sum_{s=n+1}^{\infty} \frac{b_{s} y_{s}^{\alpha+1} \frac{x_{s+1}^{\beta}-x_{s}^{\beta}}{x_{s+1}-x_{s}}}{x_{s}^{\beta} x_{s+1}^{\beta}}=\beta-\sum_{s=N}^{n} A_{s} b_{s} y_{s}^{\alpha} \frac{x_{s+1}^{\beta}-x_{s}^{\beta}}{x_{s+1}-x_{s}}
$$

since $y_{n}>0$ by (2.29), (2.30) implies

$$
\sum_{s=N}^{\infty} A_{s} b_{s} y_{s}^{\alpha} \frac{x_{s+1}^{\beta}-x_{s}^{\beta}}{x_{s+1}-x_{s}}<\infty
$$

Using the inequality $y_{n} \geq A_{n}\left(x_{n+1}^{\beta}\right)$ in (2.31) we obtain

$$
\sum_{n=N}^{\infty} b_{n} A_{n}^{\alpha+1} x_{n+1}^{\alpha \beta} \frac{x_{n+1}^{\beta}-x_{n}^{\beta}}{x_{n+1}-x_{n}}<\infty
$$


If either $\frac{x_{n+1}^{\beta}-x_{n}^{\beta}}{x_{n+1}-x_{n}}$ is nonincreasing or nondecreasing holds, then

follows. This completes the proof of the theorem.

\section{Oscillation Results}

In this section we establish criteria for all solutions of the system (1.1) to be oscillatory. First, we consider the case where the composition of functions is storngly superlinear in the sense that

$$
\int_{c}^{\infty} \frac{\mathrm{d} u}{(u)^{\alpha \beta}}<\infty
$$

and

$$
\int_{-c}^{-\infty} \frac{\mathrm{d} u}{(u)^{\alpha \beta}}<\infty \text { for all } c>0 .
$$

Theorem 3.1. Let $A_{n} \geq 0$ for $n \in N\left(n_{0}\right)$ and (3.1) hold. If

$$
\sum_{n=n_{0}}^{\infty} b_{n}\left(\left(A_{n}\right)^{\alpha}+\sum_{s=n+1}^{\infty} b_{s} A_{s}^{\alpha+1}\right)^{\alpha}=\infty \text {. }
$$

then the difference system (1.1) is oscillatory.

Proof. Assume the existence of nonoscillatory solution $\left(\left\{x_{n}\right\},\left\{y_{n}\right\}\right)$ of the system (1.1) for $n \geq N \in \mathbb{N}\left(n_{0}\right)$. As in the proof of the Theorem 2.4, we may assume that $x_{n}>0$ for all $n \geq N$. From Lemma 2.3, we have (2.22) Now following argument as in the proof of Theorem 2.5, we obtain

$$
\sum_{s=N}^{n-1} b_{s}\left(A_{s}\right)^{\alpha} \leq \sum_{s=N}^{n-1} \frac{\Delta x_{s}}{x_{s+1}^{\alpha \beta}} \leq \int_{x_{N}}^{x_{n}} \frac{\mathrm{d} u}{(u)^{\alpha \beta}}, n \geq N .
$$

Because of condition (3.1), the last inequality implies

$$
\sum_{n=N}^{\infty} b_{n}\left(A_{n}\right)^{\alpha}<\infty .
$$

Next from the second inequality (2.21), we have

$$
y_{n} \geq\left(x_{n+1}^{\beta}\right) \frac{\sum_{s=n+1}^{\infty} b_{s} A_{s}^{\alpha+1}\left(x_{s+1}^{\alpha \beta}\right)\left(x_{s+1}^{\beta}-x_{s}^{\beta}\right)}{\left(x_{s+1}-x_{s}\right) x_{s}^{\beta}}
$$

The last inequality implies

$$
y_{n} \geq\left(x_{n+1}^{\beta}\right) d \sum_{s=n+1}^{\infty} b_{s} A_{s}^{\alpha+1}, n \geq N .
$$

Again using the argument as in the proof of Theorem 2.5, we obtain

$$
d^{\alpha} \sum_{s=N}^{n-1} b_{s}\left(\sum_{i=s+1}^{\infty} b_{i} A_{i}^{\alpha+1}\right)^{\alpha} \leq \sum_{s=N}^{n-1} \frac{\Delta x_{s}}{x_{s}^{\alpha \beta}} \leq \int_{x_{N}}^{x_{n}} \frac{\mathrm{d} u}{(u)^{\alpha \beta}}
$$

for all $n \geq N$. So by condition on (3.1), we have

$$
\sum_{n=N}^{\infty} b_{n}\left(\sum_{i=n+1}^{\infty} b_{i} A_{i}^{\alpha+1}\right)^{\alpha}<\infty
$$


The inequalities (3.3) and (3.4) thus obtained clearly contradicts (3.2). This contradiction completes the proof of the theorem.

Our final result is for the case when the composition of function is strongly sublinear in the sense that

$$
\begin{aligned}
& \int_{0}^{c} \frac{\mathrm{d} u}{\left((\lambda u)^{\alpha \beta}\right)^{\alpha}}<\infty \\
& \int_{0}^{-c} \frac{\mathrm{d} u}{\left((\lambda u)^{\alpha \beta}\right)^{\alpha}}<\infty
\end{aligned}
$$

for all $c>0$ and $\lambda>0$.

Theorem 3.2. Let $A_{n} \geq 0$ for $n \in \mathbb{N}\left(n_{0}\right)$ and (3.5) hold. If

$$
\sum_{n=n_{0}}^{\infty} b_{n} A_{n}^{\alpha+1} R_{n}^{\alpha \beta}=\infty
$$

where $R_{n}=\sum_{s=n_{0}}^{n} b_{s}$, then all solutions of the system (1.1) are oscillatory.

Proof. Let $\left(\left\{x_{n}\right\},\left\{y_{n}\right\}\right)$ be a nonoscillatory solution of the system (1.1) for $n \geq N \in \mathbb{N}\left(n_{0}\right)$. As in the proof of Theorem 2.5, we may assume that $x_{n}>0$ for $n \geq N$. From the Lemma 2.3 we have (2.21). Now summing the second equation of system (1.1) from $(n+1)$ to $j$, we obtain

$$
y_{n}=y_{j}-A_{j} x_{J+1}^{\beta}+A_{n} x_{n+1}^{\beta}+\sum_{s=n+1}^{j} A_{s} b_{s} y_{s}^{\alpha} \frac{x_{s+1}^{\beta}-x_{s}^{\beta}}{x_{s+1}-x_{s}}
$$

for $j \geq n+1 \geq N$. Note that

$$
\sum_{s=n+1}^{\infty} A_{s} b_{s} y_{s}^{\alpha} \frac{x_{s+1}^{\beta}-x_{s}^{\beta}}{x_{s+1}-x_{s}}<\infty .
$$

Since otherwise it would follow from (3.9) that $y_{j}-A_{j} x_{j+1}^{\beta} \rightarrow-\infty$ as $j \rightarrow \infty$, which contradicts the first inequality of (2.21). Therefore letting $j \rightarrow \infty$ in (3.9), we obtain

$$
y_{n}=\eta+A_{n} x_{n+1}^{\beta}+\sum_{s=n+1}^{\infty} A_{s} b_{s} y_{s}^{\alpha} \frac{x_{s+1}^{\beta}-x_{s}^{\beta}}{x_{s+1}-x_{s}}, n \geq N .
$$

where

$$
\eta=\lim _{j \rightarrow \infty}\left(y_{j}-A_{j} x_{j+1}^{\beta}\right) \geq 0
$$

Define

$$
\kappa_{n}=\sum_{s=n+1}^{\infty} b_{s} A_{s}^{\alpha+1} x_{s+1}^{\alpha \beta}
$$

and in view of first inequality of (2.21) and (3.7), $\left\{\kappa_{n}\right\}$ is convergent.

From (3.11) and (3.12), we have $y_{n} \geq \lambda \kappa_{n}, n \geq N$.

Now substituting the value in the first equation of (1.1) and then summing the resulting inequality, we obtain

$$
x_{n+1} \geq x_{n} \geq \sum_{s=N}^{n-1} b_{s} \lambda \kappa_{s}^{\alpha} \geq\left(\lambda \kappa_{n-1}\right)^{\alpha} R_{n-1},
$$

Now using conditions (3.7) and (3.8) 


$$
\frac{b_{n}\left(A_{n}\right)^{\alpha+1}\left(x_{n+1}\right)^{\alpha \beta}}{\left(\left(\lambda \kappa_{n-1}\right)^{\alpha \beta}\right)^{\alpha}} \geq b_{n} A_{n}^{\alpha+1}\left(R_{n}\right)^{\alpha \beta}
$$

since $\Delta \kappa_{n-1}=-b_{n}\left(A_{n}\right)^{\alpha+1}\left(x_{n+1}\right)^{\alpha \beta}$, the above inequality can be written as,

$$
\frac{-\Delta \kappa_{n-1}}{\left(\left(\lambda \kappa_{n-1}\right)^{\alpha \beta}\right)^{\alpha}} \geq b_{n} A_{n}^{\alpha+1}\left(R_{n}\right)^{\alpha \beta}
$$

observe that for $\kappa_{n} \leq t \leq \kappa_{n-1}$, we have $\left(\left(\lambda \kappa_{n-1}\right)^{\alpha \beta}\right)^{\alpha} \geq\left((\lambda t)^{\alpha \beta}\right)^{\alpha}$, and therefore

$$
\frac{-\Delta \kappa_{n-1}}{\left(\left(\lambda \kappa_{n-1}\right)^{\alpha \beta}\right)^{\alpha}} \leq \int_{\kappa_{n}}^{\kappa_{n-1}} \frac{\mathrm{d} t}{\left((\lambda t)^{\alpha \beta}\right)^{\alpha}}
$$

Hence from (3.13) and (3.14), we obtain

$$
\int_{\kappa_{n}}^{\kappa_{N}} \frac{\mathrm{d} t}{\left((\lambda t)^{\alpha \beta}\right)^{\alpha}} \geq \sum_{s=N}^{n-1} b_{s} A_{s}^{\alpha+1} R_{s}^{\alpha \beta}
$$

which, in view of condition (3.5) and (3.8) provides a contradiction. This completes the proof of the theorem.

\section{Examples}

Example 4.1. Consider the system

$$
\begin{aligned}
& \Delta x_{n}=2.3^{5 n} y_{n}^{5} \\
& \Delta y_{n-1}=\frac{-4}{3^{n}} x_{n}^{5}
\end{aligned}
$$

Here $b_{n}=2.3^{5 n}, a_{n}=\frac{-4}{3^{n}}, y_{n}^{\alpha}=y^{5}, x_{n}^{\beta}=x_{n}^{5}$. All the necessary conditions of Theorem 3.1 are satisfied and hence the system (4.1) is oscillatory. Here, $\left(x_{n}, y_{n}\right)=\left((-1)^{n},\left\{\frac{(-1)^{n+1}}{3^{n}}\right\}\right)$ is an oscillatory solution of the system (4.1).

Example 4.2. Consider the system

$$
\begin{aligned}
& \Delta x_{n}=y_{n}^{\frac{1}{3}} \\
& \Delta y_{n-1}=\frac{-\left(4+(-1)^{n}(4 n+1)\right)}{n(n+1)} x_{n}, n \geq 1
\end{aligned}
$$

Here $\quad b_{n}=1, \quad a_{n}=\frac{-\left(4+(-1)^{n}(4 n+1)\right)}{n(n+1)}, \quad y_{n}^{\alpha}=y_{n}^{\frac{1}{3}} \quad$ and $\quad x_{n}^{\beta}=x_{n} \quad$ with $A_{n-1}=\frac{4+(-1)^{n}}{n}, n \geq 1$. we see that all conditions of Theorem 3.2 are satisfied. Hence all solutions of the system (4.2) are oscillatory.

\section{Conflicts of Interest}

The authors declare no conflicts of interest regarding the publication of this paper. 


\section{References}

[1] Agarwal, R.P. (2000) Difference Equations and Inequalities. 2nd Edition, Marcel Dekkar, New York.

[2] Castillo, S. and Pinto, M. (1998) Asymptotic Summation for Second Order Finite Difference Systems. Computers \& Mathematics with Applications, 35, 117-129. https://doi.org/10.1016/S0898-1221(97)00295-2

[3] Graef, J.R. and Thandapani, E. (1999) Oscillations of Two-Dimensional Difference Systems. Computers \& Mathematics with Applications, 38, 157-165. https://doi.org/10.1016/S0898-1221(99)00246-1

[4] Graef, J.R. and Thandapani, E. (1992) Oscillatory and Asymptotic Behaviour of Solutions of Third Order Delay Difference Equations. Funk. Ekv., 166, 272-287.

[5] Hard,y G.H., Littlewood, J.E. and Polya, G. (1988) Inequalities. 2nd Edition, Cambridge University Press, Cambridge.

[6] Lalli, B.S., Zhang, B.G. and Li, J.Z. (2001) Oscillation of Emden-Fowler Difference Systems. Journal of Mathematical Analysis and Applications, 256, 478-485. https://doi.org/10.1006/jmaa.2000.7296

[7] Li, W.T. (2001) Classification Schemes for Nonoscillatory Solutions of Two-Dimensional Nonlinear Difference Systems. Computers \& Mathematics with Applications, 42, 341-355. https://doi.org/10.1016/S0898-1221(01)00159-6

[8] Li, W.T. and Cheng, S.S. (2000) Oscillation Criteria for a Pair of Coupled Nonlinear Difference Equations. International Journal of Applied Mathematics, 2, 1327-1333.

[9] Szafranski, Z. and Szamanda, B. (1990) Oscillatoy Properties of Solutions of Some Difference Systems. Rad. Mat., 6, 205-214.

[10] Smith, B. and Taylor Jr., W.E. (1988) Nonlinear Third Order Difference Equations: Oscillatory and Asymptotic Behaviour. Tamkang Journal of Mathematics, 19, 91-95.

[11] Thandapani, E. and Ponnammal, B. (2001) On the Oscillation of a Nonlinear Two-Dimensional Difference Systems. Tamkang Journal of Mathematics, 32 201-209.

[12] Thandapani, E. and Ponnammal, B. (2000) Oscillatoy and Asymptotic Behaviour of Solutions of Two-Dimensional Difference Systems. Math. Sci. Res. Hot-Line, 4, $1-18$. 